\title{
Dynamical Model And Ride Comfort Simulation Analysis Of Distributed Drive Electric Vehicle
}

\author{
Xiao Wenwen ${ }^{1}$, Zhang Huanhuan ${ }^{1}$ \\ ${ }^{1}$ School of Automotive Engineering,Shanghai University Of Engineering Science,shanghai 201620,China
}

\begin{abstract}
This paper analyzes the ride comfort of distributed electric vehicle, simplifies a distributed electric vehicle to a fifteen degree of freedom model, and deduces the vibration differential equation by Newton 's second theorem. In this paper, a new type of hub motor vibration reduction system is established, which effectively solves the problem of large unsprung mass of distributed drive vehicles and provides a new method to improve the ride comfort of distributed drive electric vehicles. The genetic algorithm mainly regards the stiffness and damping of the suspension, hub motor damping system and tire as the design variables. The sum of root mean square value of suspension disturbance degree, body acceleration and wheel dynamic load is taken as the optimization objective function, and the limit stroke of wheel up and down and wheel dynamic load limit are taken as constraints. In order to verify the simulation optimization effect, this paper further simulates the natural frequency, damping ratio, stiffness ratio, mass ratio, speed and road surface grade of distributed drive electric vehicle. The results show that the optimization of the stiffness and damping of distributed-drive electric vehicles effectively improves ride comfort and passenger comfort. The 15 DOF model of distributed electric vehicle provides a theoretical basis for analyzing the ride comfort of distributed electric vehicle.
\end{abstract}

\section{Introduction}

Due to the continuous energy consumption, electric vehicles have become an important research direction for energy conservation.the driving mode of electric vehicles has a centralized arrangement, wheel drive and distributed drive.At present, the use of distributed driver has become the focus of current research.However, the large unsprung mass of distributed drive electric vehicles will inevitably affect the ride comfort of electric vehicles. Nagaya[1] pointed out that the wheel hub motor is directly connected with the wheel, the impact of the motor is obvious on the ground, the life of the motor has a tremendous impact.the drive system of distributed drive electric vehicle is designed by the method of converting the hub motor suspension into vibration absorber. Hrovat[2] pointed out that due to the introduction of wheel hub motors, distributed drive electric vehicles significantly increase the unsprung mass, so that the suspension of the dynamic load, wheel load and acceleration of the vehicle body root mean square significantly increased. Rmsden[3] describes the integrated design of the motor and other parts of the tire to find ways to improve vertical performance. Shino[4] established a two-freedom model of the micro-distributed drive electric vehicle to realize the direct control of the yaw moment by distributing the driving force and the braking force so as to improve the handling stability of the vehicle. Esmailzadeh[5] established a four-wheel drive eight-degree-of-freedom vehicle dynamics model.
Some domestic researchers to improve the ride comfort of distributed electric vehicle research.Guobao Ning[6] analyzed the unsprung mass of distributed electric vehicles on the ride has a greater impact on the ride, and gives the wheel hub motor suspension model simulation results. Xiacun, Liang[7] used to control the active suspension to solve the problem of non-sprung mass.Rui,Liang[8] pointed out the use of dynamic vibration absorber to improve the unsprung mass problem, the results show that the wheel relative dynamic load and body vibration acceleration significantly reduced.Yane,Zhao[9] pointed out that the stator mass of the motor is transformed into a sprung mass that can effectively lower the highest peak of the vibration acceleration of the body. Based on the current driving modes of various electric vehicles, the author establishes the linear vibration model and dynamic differential equation of a 15-DOF distributed electric vehicle.Passenger ride comfort is evaluated by solving the weighted root mean square acceleration value. to the body acceleration, suspension disturbance and wheel dynamic load as the optimization goal. optimize system parameters such as suspension damping and suspension stiffness and damping on the motor[10].

\section{Model Establishment}

\subsection{Vehicle dynamics model}


Distributed drive electric vehicle is a multi-degree of freedom nonlinear system composed of many components. The coupling of its subsystems makes the whole system of the vehicle more complicated[11]. The study found that when the lateral acceleration is generally not more than $0.4 \mathrm{~g}$, it can be considered that the vehicle simplified model is a linear system[12]. The following assumptions need to be made before simplifying the model: (1) Hypothesis road roughness coefficient unchanged, only with the selected pavement level; (2) The car is doing a uniform linear motion, regardless of its turning and acceleration of the situation; (3) The wheel hub motor output power remains unchanged at different road surface levels; (4) The same is true of the road surface that is entered for the four wheels; (5) All parts in the car are considered as rigid bodies and the mass suspension distribution coefficient has a value of one; (6) Suspension, tire stiffness and damping constant; (7) Uneven road surface roughness process is a steady state random process[13]. simplify the following 15 degrees of freedom for distributed drive electric vehicles. Body pitch, roll, yaw, vertical vibration of the four wheels, Vertical vibration of four wheel-connected hub motors and vertical displacement of one driver and three passengers for a total of 15 degrees of freedom. Simplified vehicle dynamics model, shown in Figure 1.

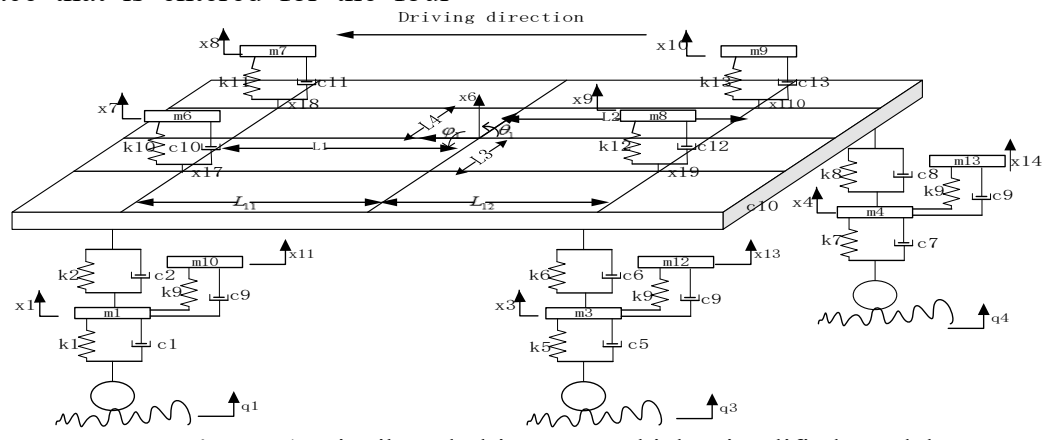

Figure 1 Distributed drive EV vehicle simplified model

Suspension and body connection position in the vertical displacement:

$\left\{\begin{array}{l}x_{11}=x_{6}-a \theta_{1}+B \varphi_{1} \\ x_{12}=x_{6}-a \theta_{1}-B \varphi \\ x_{13}=x_{6}+a \theta_{1}+B \varphi_{1} \\ x_{14}=x_{6}+a \theta_{1}-B \varphi_{1}\end{array}\right.$

Seat and body connection position in the vertical displacement:

$\left\{\begin{array}{l}x_{17}=x_{5}-L_{1} \theta_{1}+L_{3} \varphi_{1} \\ x_{18}=x_{5}-L_{1} \theta_{1}-L_{3} \varphi_{1} \\ x_{19}=x_{5}+L_{1} \theta_{1}+L_{3} \varphi_{1} \\ x_{110}=x_{5}+L_{1} \theta_{1}-L_{3} \varphi_{1}\end{array}\right.$

Four non-sprung mass vertical vibration differential equations:

$\left\{\begin{array}{l}m_{1} \ddot{x}_{1}+k_{9}\left(x_{1}-x_{5}\right)+c_{9}\left(\dot{x}_{1}-\dot{x}_{5}\right)+k_{2}\left(x_{1}-x_{11}\right) \\ +c_{2}\left(\dot{x}_{1}-\dot{x}_{11}\right)+k_{1}\left(x_{1}-q_{1}\right)+c_{1}\left(\dot{x}_{1}-\dot{q}_{1}\right)=0 \\ m_{5} \ddot{x}_{5}+k_{9}\left(x_{5}-x_{1}\right)+c_{9}\left(\dot{x}_{5}-\dot{x}_{1}\right)=0 \\ m_{2} \ddot{x}_{2}+k_{9}\left(x_{2}-x_{5}\right)+c_{9}\left(\dot{x}_{2}-\dot{x}_{5}\right)+k_{4}\left(x_{2}-x_{12}\right) \\ +c_{4}\left(\dot{x}_{2}-\dot{x}_{12}\right)+k_{3}\left(x_{2}-q_{2}\right)+c_{3}\left(\dot{x}_{2}-\dot{q}_{2}\right)=0 \\ m_{4} \ddot{x}_{4}+k_{9}\left(x_{4}-x_{5}\right)+c_{9}\left(\dot{x}_{4}-\dot{x}_{5}\right)+k_{8}\left(x_{4}-x_{14}\right) \\ +c_{8}\left(\dot{x}_{4}-\dot{x}_{14}\right)+k_{7}\left(x_{4}-q_{4}\right)+c_{7}\left(\dot{x}_{4}-\dot{q}_{4}\right)=0\end{array}\right.$

Body center of mass vertical vibration differential equation:

$$
\begin{aligned}
& m_{1} \ddot{x}_{6}+k_{2}\left(x_{11}-x_{1}\right)+k_{4}\left(x_{12}-x_{2}\right)+k_{6}\left(x_{13}-x_{3}\right) \\
& +k_{8}\left(x_{14}-x_{4}\right)+k_{10}\left(x_{17}-x_{7}\right)+k_{11}\left(x_{18}-x_{8}\right) \\
& +k_{12}\left(x_{19}-x_{9}\right)+k_{13}\left(x_{110}-x_{10}\right)+c_{2}\left(\dot{x}_{11}-\dot{x}_{1}\right) \\
& +c_{4}\left(\dot{x}_{12}-\dot{x}_{2}\right)+c_{6}\left(\dot{x}_{13}-\dot{x}_{3}\right)+c_{8}\left(\dot{x}_{14}-\dot{x}_{4}\right) \\
& +c_{10}\left(\dot{x}_{17}-\dot{x}_{7}\right)+c_{11}\left(\dot{x}_{18}-\dot{x}_{8}\right)+c_{12}\left(\dot{x}_{19}-\dot{x}_{9}\right) \\
& +c_{13}\left(\dot{x}_{110}-\dot{x}_{10}\right)=0
\end{aligned}
$$

According to the formula (1) - (7) can get people and vehicles system 15 degrees of freedom and more mechanical equations:

$$
M \ddot{X}+C \dot{X}+K X=C_{t} Q+K_{t} Q
$$

Where $\mathrm{M}$ is the mass matrix, $\mathrm{C}$ is the damping matrix, and $\mathrm{K}$ is the stiffness matrix. $K_{t}, C_{t}$ Enter the stiffness and damping matrix, $\mathrm{X}$ is the displacement of the column vector, $Q$ is the road surface random excitation. The specific expression is as follow.

\subsection{Road excitation model}

When driving distributed electric vehicles, different speed and road surface levels should be considered. Different road input incentives, need to establish a different random road.

Pavement incentives to establish the main methods are the following. Harmonic superposition method, trigonometric series method, white noise filtering method and wavelet analysis. The triangular series method is through a large number of random phase sine or cosine functions combined into a random road excitation[14]. 


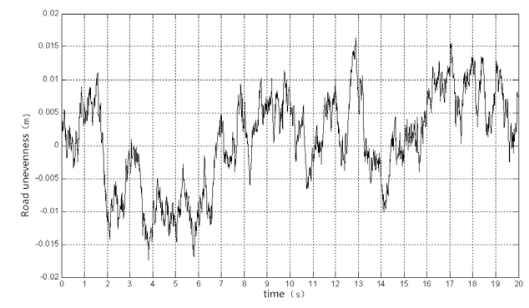

Figure 2 Right front road surface excitation

\section{Basic Evaluation Methods}

According to matlab simulation analysis of the human-seat system in the axial weighted acceleration $\operatorname{rms} a_{w}$. Through the corresponding frequency weighting function $w(f)$. Acceleration time history is obtained by filtering the network $a_{w}(t)$, And by the time-domain integration method can be obtained by the following formula rms value $a_{w}{ }^{[16]}$.

$$
a_{w}=\left[\frac{1}{T} \int_{0}^{T} a_{w}{ }^{2}(t) d t\right]^{\frac{1}{2}}
$$

Spectrum analysis of the acceleration time to obtain the power spectral density function, get the specific weighted acceleration $\operatorname{rms}$ value $a_{w}$. As the following formula shows:

$$
a_{w}=\left[\int_{0.5}^{80} w^{2}(f) G_{a}(f) d f\right]^{\frac{1}{2}}
$$

The rms value of the total weighted acceleration requires the sum of the rms values of the various axial weighted accelerations, The summation formula is as follows:

$$
a_{v}=\left[\left(1.4 a_{x w}\right)^{2}+\left(1.4 a_{y w}\right)^{2}+a_{z w}{ }^{2}\right]^{\frac{1}{2}}
$$

\section{Genetic Algorithm Simulation Optimizatio Analysis.}

\subsection{Overview of Genetic Algorithms}

The genetic algorithm (Genctic Algorithm) is an adaptive global optimization probabilistic search method that simulates the process of the organism's genetic and evolutionary survival in the natural environment. Originally proposed by John Holland, a computational model of solving optimization problems by simulating natural evolutionary processes ${ }^{[17]}$. The computation of genetic algorithms is similar to that of nature ${ }^{[18]}$. The use of genes on chromosomes to find eligible chromosomes addresses practical problems.Genetic algorithms are similar to those in nature. Genetic algorithms are not known to solve any problem. They just correctly evaluate the chromosome generated by the algorithm and determine the chromosome according to the fitness value. Make better chromosomes have better breeding opportunities.

\subsection{Genetic Algorithm Operation}

Before starting the calculation, $\mathrm{n}$ random individuals need to be generated. These random individuals form an initial population for genetic algorithm search, and then $n$ individuals are encoded. It is necessary to calculate the fitness of $\mathrm{n}$ individuals and then select crossover and mutation of individuals with larger fitness. Then calculate the fitness of each individual, retain the individuals with large fitness, as the next generation of populations. After many iterations, the individuals with the highest fitness value are obtained. The genetic algorithm used in this paper is the proportion of choice, a single point of intersection and basic bit variation composed of three algorithms. Genetic algorithm parameters set as follows:The population size is 200 , the number of termination iterations is 300 , the crossover probability is 0.9 , the mutation probability is 0.0001 and the discreteness is 0.001 .

\subsection{The objective function}

The purpose of optimization is to adjust the stiffness of the seat and the damping of the seat by adjusting the springs of the damping system between the tire, suspension, motor and wheel. Improve the smoothness of distributed electric vehicle and passenger comfort.Taking the sum of root mean square values of body acceleration, wheel dynamic load and suspension disturbance as optimization objective function:

$$
J_{\text {min }}=\sigma_{\ddot{x}}+\sigma_{f d}+\sigma_{F d / G}
$$

Body acceleration, wheel dynamic load and suspension disturbance are as follows:

$$
\begin{aligned}
& \sigma_{\ddot{x}}=\sqrt{4 \pi G_{q}\left(n_{0}\right) n_{0}{ }^{2} u \int_{0}^{\infty}|H(j w)|_{\dot{x} \sim \dot{q}}^{2} d f} \\
& \sigma_{F d / G}=\sqrt{4 \pi G_{q}\left(n_{0}\right) n_{0}{ }^{2} u \int_{0}^{\infty}|H(j w)|_{F d / G \sim \dot{q}}^{2} d f} \\
& \sigma_{f d}=\sqrt{4 \pi G_{q}\left(n_{0}\right) n_{0}{ }^{2} u \int_{0}^{\infty}|H(j w)|_{f d \sim \dot{q}}^{2} d f}
\end{aligned}
$$

\subsection{Design Variables}

Selecting as the hub motor and the wheel damping system between the spring stiffness $k_{9}$, Damping coefficient $c_{9}$; The stiffness of the wheel $k_{1}$, Damping coefficient $c_{1}$; Stiffness of the suspension $k_{2}$, Damping coefficient $c_{2}$; The stiffness of the damping system between the seat and the bodywork $k_{10}$,Damping coefficient $c_{10}$;

\subsection{Constraints}

In order to make the Distributed drive electric run normally, it is necessary to maintain suspension disturbance and wheel dynamic load in proper range. According to the car theory, it can be seen that the suspension disturbance degree and wheel dynamic load should meet the following constraints: 


$$
\left\{\begin{array}{l}
3 \sigma_{f d} \leq\left[f_{d}\right] \\
3 \sigma_{F d / G} \leq 1
\end{array}\right.
$$

In the formula, $\sigma_{f d}$-Suspension disturbance RMSE value; $\left[f_{d}\right]$-Suspension suspension with the degree of value; $\sigma_{F d / G}$-Wheel relative dynamic load;

\subsection{Optimization Results Analysis}

Table 1 vehicle parameters

\begin{tabular}{|c|c|}
\hline Vehicle parameters symbol & $\begin{array}{c}\text { Vehicle parameter } \\
\text { value }\end{array}$ \\
\hline$m_{1}, m_{2}, m_{3}, m_{4}(\mathrm{~kg})$ & 45 \\
\hline$m_{10} m_{11} m_{12} m_{13}(\mathrm{~kg})$ & 24 \\
\hline
\end{tabular}

Table 2 before and after optimization parameters

\begin{tabular}{|c|c|c|}
\hline parameter & Initial value & Optimization value \\
\hline$k_{1}(N / m)$ & 20000 & 15000 \\
\hline$c_{1}(N \bullet s / m)$ & 100 & 50 \\
\hline$k_{2}(N / m)$ & 17000 & 13000 \\
\hline$c_{2}(N \bullet s / m)$ & 1215 & 1100 \\
\hline$k_{9}(N / m)$ & 72500 & 70000 \\
\hline$c_{9}(N \bullet s / m)$ & 3020 & 2800 \\
\hline$k_{10}(N / m)$ & 8100 & 7500 \\
\hline$c_{10}(N \bullet s / m)$ & 1000 & 815 \\
\hline
\end{tabular}

By Matlab and adams software co-simulation, analysis of distributed electric vehicles before and after optimization of the changes in the smooth. Optimization as shown below.

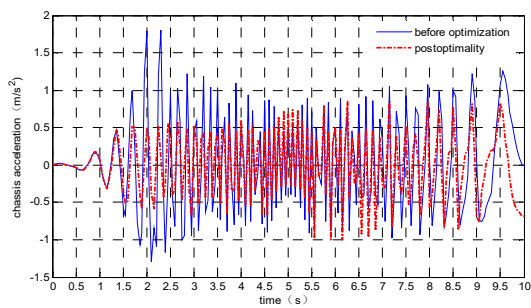

Figure 5 body acceleration

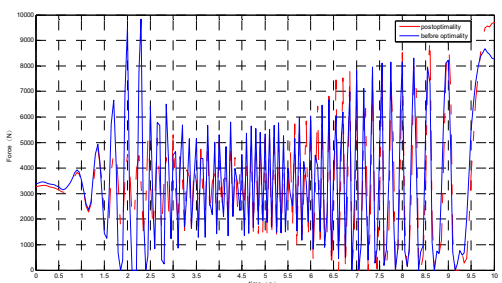

Figure 6 Wheel dynamic load

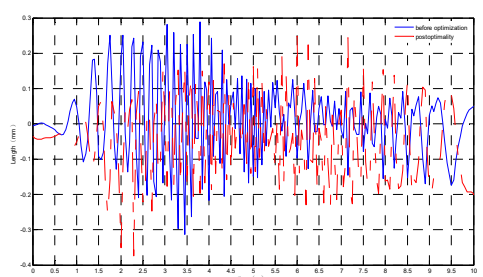

Figure 7 Suspension disturbance

It can be seen from Figure 5 that the distributed driving electric vehicle can reduce the vehicle body acceleration by $20 \%$ after optimization through the ride comfort simulation test, and improve the vehicle ride comfort.It can be seen from Figure 6 that the optimized suspension stiffness and damping have little effect on the wheel dynamic load. It can be seen from Figure 7 that the disturbance degree of the suspension after optimization is obviously improved, and the suspension disturbance degree is reduced by about $15 \%$. In summary, the optimized stiffness and damping can effectively improve the ride comfort of distributed electric vehicles.

\section{Conclusion}

1)This paper establishes a 15 -freedom model, which can provide a theoretical basis for studying the ride comfort of distributed-drive electric vehicles and better study on the ride-comfort of distributed-drive electric vehicles.

2)In this paper, we get the best suspension stiffness and damping, the stiffness and damping of the vibration damping system between the wheel and the hub motor, and the damping of the tire by genetic algorithm. Identify the more favorable stiffness and damping for the ride comfort of distributed electric vehicles.

3)In order to further analyze the influence of stiffness and damping after optimization on the distributed electric vehicle, the relationship between different driving speeds and passenger comfort is obtained. It can be seen from the table that within $90 \mathrm{~km} / \mathrm{h}$, passenger comfort is very good.

4)In addition, the influence of the natural frequency, damping ratio, stiffness ratio and mass ratio of the distributed electric vehicle on the ride comfort is further analyzed. The results show that the appropriate principles should be followed in the selection of these influencing factors.

\section{Biographical notes}

Wen wen Xiao, born in 1993, is currently a master candidate at Automotive Engineering Institute. Shanghai University Of Engineering Science, China.

Tel: +86-13386206059;E-mail: axiaowwa@163.com.

Huan huan Zhang, born in 1979, is currently an professor at Shanghai University of Engineering Science, China. She received his $\mathrm{PhD}$ degree from jilin Universtiy, China, in 2009. Her research interests include Electric vehicle key technology. E-mail:zhanghh@sues.edu.cn

\section{References}

1. Naga G.Development of an in-Wheel drive with 
advanced dynamic-damper mechanism. JSEA Review, 2003, 24 (4): 477-481.

2. Hrovat D.Influence of Unsprung Weight on Vehicle Ride Quality.Journal of Sound and Vibration. 1988, 124(3): 497-516.

3. Resden V S R. Design of an in-Wheel Motor for a Solar Powered Electric Vehicle. Journal\&Magazines, 1998, 145(5): 402-408.

4. Shino M, Nagai M. Yaw-moment control of electric vehicle for improving handing and stability. JSAE review, 2001, 22(04): 473-480.

5. Esmailzadeh E, Vossoughi G R, Goodarz A. Dynamic modeling and analysis of a four motorized wheel electric vehicle. Vehicle system dynamics, 2001, 35(03): 163-194.

6. G B Ning, G Wan. The Present Research Situation of the Influences on Vehicle Vertical Performances Induced by Direct Wheel Drives System. Automobile Technology, 2007(3): 21-25.

7. C L Xia, G B Ning. Active control of vertical vibration negative influences induced by high unsPrung mass of in-wheel motor electric vehicle. Chinese Journal of Construction Machinery, 2006, 4(01): 31-42,42.

8. R Liang, Z P Yu, G B Ning. Restraint of negative effect on vertical vibration of wheel rim driven motor vehicle based on shake absorption principle. Journal of Machine Design, 2008, 25(01): 28-30

9. Y E Zhao, J W Zhang, X Han. Design and study on the dynamic damper mechanism for an In-wheel motor individual drive electric vehicle. Mechanical Science and Technology for Aerospace Engineering, 2008, 27(03): 395-398.

10. Y Ma, Z X Deng, D Xie. Analysis and optimization of in-wheel motor suspension configureation. Journal of Central South University(Science and Technology, 2014, 45(09): 3008-3014.

11. G X Zhang, D Ye.Eleven DOF vehicle dynamics model and comfort simulation. Machinery Design \& Manufacture,2017(01):43-46.

12. Z G Huang, H Zhu, X C Liang, E R Mao. Study on dynamic simulation of subminiature car between model of seven DOF and eight DOF. Computer Simulation, 2011, 28(2): 354-359.

13. $\mathrm{X} \mathrm{H}$ Mo, $\mathrm{Y} H$ Zhao, $\mathrm{Z} H$ Zhong, $\mathrm{Y}$ Zhang.Robustness optimization of ride comfort for vehicle based on $6 \sigma$ method. Journal of Central South University (Science and Technology), 2012, 43(11): 4286-4292.

14. D Z Xu, Z F Zhang, G C Xia. Research on simulation method of vehicle pavement roughness excitation. Journal of Graphics. 2016, 37(05): 668-674.

15. X P Wang, W B Liu, J J Huang, Z Y Li. Simulation analysis of vehicle vibration under randomly road excitation. Journal of shandong jiaotong universtiy, 2010, 18(3): 7-11.

16. Z S Yu. Automobile Theory. 5th edn.China Machine
Press.Beijing,China.

17. Q Zhao, H J Che,J Li. Automobile ride optimization simulation based on genetic algorithm. Machinery Design \& Manufacture,2009(03): 203-205

18. G Y Pan, H L Yu. optimization of suspension dynamic absorbers based on genetic algorithm. Manufacturing Automation, 2014, 36(20): 82-84. 